\title{
$\mathrm{VCM}$ 을 이용한 6자유도 능동형 제진시스템 개발
}

\section{Development of a 6-DOF Active Vibration Isolation System Using Voice Coil Motor}

\author{
길 형 균†. 김 광 산* \\ Hyeong Gyeun Gil and Kwang San Kim \\ (2010년 4월 1일 접수 ; 2010년 6월 18일 심사완료)
}

Key Words : Active Isolation System(능동형 제진시스템), Voice Coil Motor(보이스코일모터), Transmissibility (전달률), Loop Shaping(루프세이핑), AFM(원자힘현미경)

\begin{abstract}
The paper is about the development of 6-DOF active vibration isolation systems using VCM. Firstly, formulate the vertical 3-DOF mathematical model under eccentric load, and compare the model with the case in which the center of mass is located at the centroid. And then, complete the 6-DOF mathematical model by formulating the horizontal 3-DOF mathematical model. Find main parameters by comparing the result of the frequency response test with simulation result on the model. Finally, achieve the performance of vibration isolation by applying loop shaping approach \& feedforward controller.
\end{abstract}

\section{기 호 설 명}

$B$ : 입력 계수행렬

$C$ : 감쇠 계수행렬

$F_{d}, F_{d d}:$ 외란 계수행렬

$I_{x}, I_{y}, I_{z}$ : 질량관성모멘트

$K$ : 강성 계수행렬

$l$ : 질량중심에서 스프링까지의 거리

$M$ : 질량 계수행렬

\section{1. 서 론}

나노수준에서 소재의 형상이나 구조, 특성들을 측 정하기 위하여 다양한 종류의 원자현미경들을 연구 및 산업분야에서 널리 사용하고 있다. 그중에서도

† 교신저자; 정회원, (주)대일시스템

E-mail : waycon67@empal.com

Tel : (031)339-3375, Fax : (031)339-3376

* (주)대일시스템 원자힘현미경(atomic force microscope: $\mathrm{AFM}$ )은 주사터널현미경(STM)의 결점이었던 부도체 시료에 대한 측정능력을 보완함으로써 그 활용범위 및 시장 규모가 크게 확대되고 있다. $\mathrm{AFM}$ 이 올바르게 동작 하며 나노레벨의 정밀한 측정을 수행하기 위해서는 환경 소음을 차단하고, 장비 자체의 진동모드들을 분 석하여 제거해야만 한다. 또한 바닥으로부터 전달되 는 진동외란을 효과적으로 차단시킬 수 있어야 원하 는 성능을 발휘할 수 있기 때문에 능동형 제진시스 템(active vibration isolation system: AVIS)의 사용은 필수적이다.

AFM용 AVIS는 메탈스프링의 수동소자와 piezo electric transducer(PZT) 또는 보이스 코일 모터 (voice coil motor: $\mathrm{VCM}$ )등의 능동소자를 직/병렬 로 결합하여 제진성능을 구현하고 있다. 특히 $\mathrm{VCM}$ 은 빠른 응답특성, 제작의 용이성 등의 장점이 있어 다양하게 연구 및 응용되고 있다. 예로써 나노 인덴 터 용으로 조주희 등은 ${ }^{(1)}$ 형상 최적화를 통한 $\mathrm{VCM}$ 의 최적설계를, 이홍교 등은 ${ }^{(2)}$ 추력부와 분해능 향상을 
위한 부분으로 분리된 2중구조 원통형 $\mathrm{VCM}$ 설계 를 통하여 제품에 응용하였으며, 최기봉 등은 ${ }^{(3)}$ 공 심솔레노이드와 영구자석사이의 힘의 상관관계를 해석한 후 이를 미소위치결정기구의 시스템 설계에 반영한 연구결과를 발표한 바 있다.

현재 AFM용 AVIS시장을 점유하고 있는 대표적 인 제품 중 하나는 독일 Halcyonics사의 micro series 로서 메탈스프링과 $\mathrm{VCM}$ 이 병렬로 결합되어 있다. $\mathrm{VCM}$ 의 구조는 고정자와 운동자가 membrane으로 연결되는 방식으로 muller ${ }^{(4)}$ 등의 논문에 잘 나타나 있다. 그들은 논문에서 질량중심이 도심에 위치한다 는 가정에서 3 자유도 시스템에 대한 수학적 모델을 수립하고 실험에 기초하여 파라미터 값들을 추정하 였다. 또한 아날로그 제어기를 통하여 $10 \mathrm{~Hz}$ 에서 roll-off rate $-40 \mathrm{~dB}$ 성능을 실현하고 있다.

AVIS의 시스템 구성은 대부분 유사하여 제품의 우수성은 사용된 수동 및 능동소자의 특성과 제어성 능에 크게 의존한다. 문준희 등은 ${ }^{(5)}$ 레버 링키지를 이용한 시스템구성에 대한 연구를 수행한 바 있다. 그리고 성능과 관련하여 feedforward 제어 및 loop shaping 설계기법을 응용할 경우 상대적으로 좋은 제 진성능을 나타내는 것으로 보고되고 있다 ${ }^{(4,6,7)}$.

따라서 이 연구에서는 편심상태에서의 수직방향 3 자유도 수학적 모델을 수립하여 질량중심이 도심에 위치할 때의 결과와 비교·검토한 후 이를 수평방향 으로 확장하여 6자유도 시스템 모델링을 완성한다. 또한 loop shaping 설계개념 및 feedforward 제어기법 을 응용하여 $10 \mathrm{~Hz}$ 에서의 roll-off rate $-40 \mathrm{~dB}$ 성능을 구현하고자 한다.

\section{2. 시스템 구성}

Fig. 1에 나타낸 능동형 제진시스템은 상-하 플레 이트 사이에 테이퍼 형태의 압축코일스프링이 설치 되어 상부 하중을 지지하고, 바닥에서 올라오는 진동 의 일부를 차단한다. 능동소자로써 스프링과 병렬로 위치한 비접촉식 $\mathrm{VCM}$ 을 사용하고 있으며, 진동속도 검출을 위하여 geospace사의 GS-11D geophone 센 서를 사용하였다. geophone 센서는 공진주파수가 $4.5 \mathrm{~Hz}$ 인근에 있기 때문에 그 이하의 주파수영역 은 신호처리를 통한 보상이 필요하다. 이 연구에서는 디지털 필터를 설계하여 이러한 문제를 해결하였다.

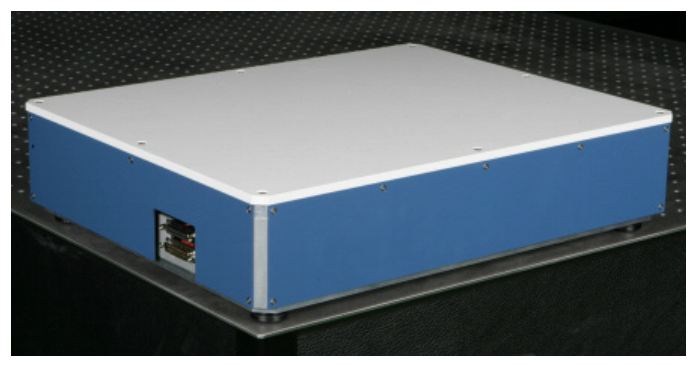

Fig. 1 6-DOF active vibration isoaltion system

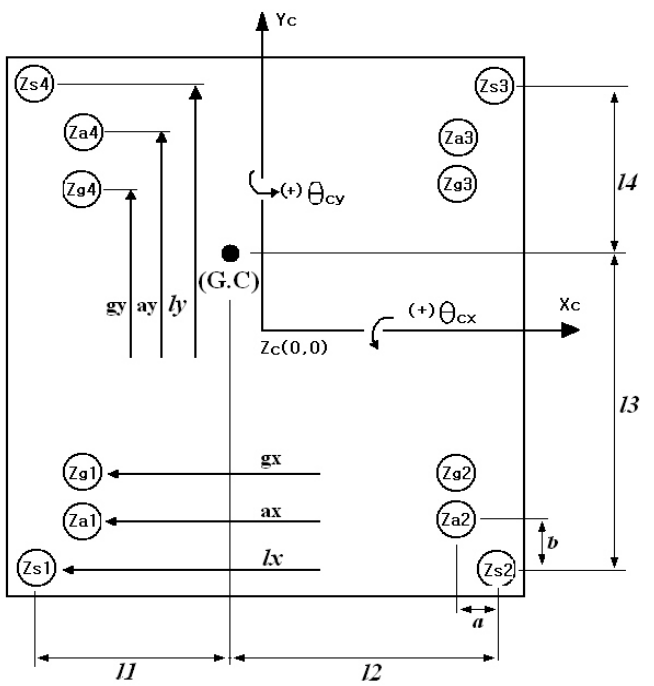

Fig. 2 Layout of vertical components

그리고 제어알고리듬 개발 및 각종 시험들은 독일의 $\mathrm{dSPACE}$ 장비를 이용하여 실시하였다.

\section{3. 수학적 모델}

운동방정식의 유도를 위해 각각의 변위크기는 작 고, 신호 검출은 스프링 체결 지점에서 이루어진다 고 가정한다 ${ }^{(8)}$.

\section{1 수직방향 운동방정식}

바닥에서 전달된 변위 $d(t)$ 에 의한 상부 플레이트 의 $\mathrm{z}$ 축 변위에 대하여 3 자유도 운동방정식은 수직 방향 직선운동(heaving), $\mathrm{x}$ 축을 중심으로 한 회전운 동(pitching), $\mathrm{y}$ 축을 중심으로 한 회전운동(rolling)으 로 각각 유도할 수 있으며, 각 구성품의 배치상태 및 좌표를 Fig. 2에 나타내었다. 
각 방향에 대한 변위를 $z_{c}(t), \theta_{x}(t), \theta_{y}(t)$ 라 하 고, 각 스프링 위치에서의 수직방향 변위를 나타내 면 아래와 같다.

$$
\begin{aligned}
& z_{1}(t)=z_{c}-l_{3} \theta_{x}(t)+l_{1} \theta_{y}(t) \\
& z_{2}(t)=z_{c}-l_{3} \theta_{x}(t)-l_{2} \theta_{y}(t) \\
& z_{3}(t)=z_{c}+l_{4} \theta_{x}(t)-l_{2} \theta_{y}(t) \\
& z_{4}(t)=z_{c}+l_{4} \theta_{x}(t)+l_{1} \theta_{y}(t)
\end{aligned}
$$

각 스프링 변위로부터 질량중심에서의 변위로 식 (1)을 변환하면 아래와 같다.

$$
\begin{aligned}
& z_{c}(t)=\frac{1}{4}\left(z_{1}+z_{2}+z_{3}+z_{4}\right) \\
& \theta_{x}(t)=\frac{1}{2 l_{y}}\left(-z_{1}-z_{2}+z_{3}+z_{4}\right) \\
& \theta_{y}(t)=\frac{1}{2 l_{x}}\left(z_{1}-z_{2}-z_{3}+z_{4}\right)
\end{aligned}
$$

운동방정식은 바닥으로부터 전달되는 외란입력과 액추에이터 제어입력을 포함시켜 전개한 후 이를 정리하면 식 (3)과 같다. 이때 각 스프링 상수 $k$ 및 감쇠상수 $c$ 는 모두 동일하다고 가정한다.
Table 1 Estimated parameter values for vertical direction

\begin{tabular}{c|c|c|c}
\hline \hline Parameter & Value & Parameter & Value \\
\hline$m_{c}$ & $22.5[\mathrm{~kg}]$ & $f_{z_{c}}$ & $5.8[\mathrm{~Hz}]$ \\
\hline $\mathrm{c}$ & $2[\mathrm{Ns} / \mathrm{m}]$ & $f_{\theta_{x}}$ & $8.9[\mathrm{~Hz}]$ \\
\hline $\mathrm{k}$ & $7387.3[\mathrm{~N} / \mathrm{m}]$ & $f_{\theta_{y}}$ & $8.5[\mathrm{~Hz}]$ \\
\hline$l_{1}$ & $0.21[\mathrm{~m}]$ & $l_{2}$ & $0.23[\mathrm{~m}]$ \\
\hline$l_{3}$ & $0.275[\mathrm{~m}]$ & $l_{4}$ & $0.265[\mathrm{~m}]$ \\
\hline$R$ & $16.6[\Omega]$ & $k_{f}$ & $4.2[\mathrm{~N} / \mathrm{V}]$ \\
\hline$I_{x}$ & $0.6889\left[\mathrm{~kg} \mathrm{~m}^{2}\right]$ & $I_{y}$ & $0.5014\left[\mathrm{~kg} \mathrm{~m}^{2}\right]$ \\
\hline
\end{tabular}

$$
\begin{gathered}
\text { 여기서, } \bar{x}=\left[z_{c}(t), \theta_{x}(t), \theta_{y}(t)\right]^{T} \\
v(t)=\left[v_{1}(t), v_{2}(t), v_{3}(t), v_{4}(t)\right]^{T} \\
d(t)=\left[d_{1}(t), d_{2}(t), d_{3}(t), d_{4}(t)\right]^{T} \\
\dot{d}(t)=\left[\dot{d}_{1}(t), \dot{d}_{2}(t), \dot{d}_{3}(t), \dot{d}_{4}(t)\right]^{T} \text { 이다. }
\end{gathered}
$$

식 (3)에서 $v(t)$ 는 시스템에 대한 $\mathrm{VCM}$ 의 전압입 력이고, $d(t)$ 는 바닥에서 전달되는 외란 입력항이 다. 그리고 $\mathrm{VCM}$ 의 인덕턴스 $(L=3.1 \mathrm{mH})$ 와 유기전 압 상수 $\left(K_{e}\right)$ 는 크기가 비교적 작아 무시하였다.

외란이 없다는 가정하에서 $0.1 \mathrm{~Hz}$ 에서부터 100 $\mathrm{Hz}$ 까지의 $0.1 \mathrm{~V}$ 전압진폭의 sweep-sine 신호를 $\mathrm{z} 1$

$$
\begin{aligned}
& M \ddot{\bar{x}}+D \dot{\bar{x}}+\overline{K \bar{x}}=B v(t)+F_{d} d(t)+F_{d d} \dot{d}(t) \\
& M=\left[\begin{array}{ccc}
m & 0 & 0 \\
0 & I_{x} & 0 \\
0 & 0 & I_{y}
\end{array}\right] \\
& D=\left[\begin{array}{ccc}
4 c & -2 c\left(l_{3}-l_{4}\right) & 2 c\left(l_{1}-l_{2}\right) \\
-2 c\left(l_{3}-l_{4}\right) & 2 c\left(l_{3}^{2}+l_{4}^{2}\right) & -c\left(l_{13}-l_{23}+l_{24}-l_{14}\right) \\
2 c\left(l_{1}-l_{2}\right) & -c\left(l_{13}-l_{23}+l_{24}-l_{14}\right) & 2 c\left(l_{1}^{2}+l_{2}^{2}\right)
\end{array}\right] \\
& K=\left[\begin{array}{ccc}
4 k & -2 k\left(l_{3}-l_{4}\right) & 2 k\left(l_{1}-l_{2}\right) \\
-2 k\left(l_{3}-l_{4}\right) & 2 k\left(l_{3}^{2}+l_{4}^{2}\right) & -k\left(l_{13}-l_{23}+l_{24}-l_{14}\right) \\
2 k\left(l_{1}-l_{2}\right) & -k\left(l_{13}-l_{23}+l_{24}-l_{14}\right) & 2 k\left(l_{1}^{2}+l_{2}^{2}\right)
\end{array}\right] \\
& B=\left[\begin{array}{cccc}
\frac{k_{f 1}}{R_{1}} & \frac{k_{f 2}}{R_{2}} & \frac{k_{f 3}}{R_{3}} & \frac{k_{f 4}}{R_{4}} \\
-\left(l_{3}-b\right) \frac{k_{f 1}}{R_{1}}-\left(l_{3}-b\right) \frac{k_{f 2}}{R_{2}} & \left(l_{4}-b\right) \frac{k_{f 3}}{R_{3}} & \left(l_{4}-b\right) \frac{k_{f 4}}{R_{4}} \\
\left(l_{1}-a\right) \frac{k_{f 1}}{R_{1}} & -\left(l_{2}-a\right) \frac{k_{f 2}}{R_{2}}-\left(l_{2}-a\right) \frac{k_{f 3}}{R_{3}}\left(l_{1}-a\right) \frac{k_{f 4}}{R_{4}}
\end{array}\right] \\
& F_{d}=\left[\begin{array}{cccc}
k & k & k & k \\
-k l_{3}-k l_{3} & k l_{4} & k l_{4} \\
k l_{1}-k l_{2}-k l_{2} & k l_{1}
\end{array}\right] \quad F_{d d}=\left[\begin{array}{cccc}
c & c & c & c \\
-c l_{3}-c l_{3} & c l_{4} & c l_{4} \\
c l_{1}-c l_{2}-c l_{2} & c l_{1}
\end{array}\right]
\end{aligned}
$$


액추에이터에 입력하여 주파수응답시험을 수행하였 다. 시험결과로부터 미지의 파라미터 값들은 추정하 였으며 Table 1과 같다. 여기서 VCM 코일의 내부 저항은 측정값이고, 힘상수에 해당하는 $k_{f}$ 는 전압과 힘과의 관계를 나타내며 시험결과로부터 추정한 값 이다.

식 (3)에 대하여 상태변수를 $\left[x_{1}, x_{2}, x_{3}, x_{4}, x_{5}, x_{6}\right]^{T}=$ $\left[z_{c}, \theta_{x}, \theta_{y}, \dot{z}_{v} \dot{\theta}_{x}, \dot{\theta}_{y}\right]^{T}$ 로 정의하고, 상태공간 표현식을 유도하면 아래와 같다.

$$
\begin{aligned}
& \dot{x}(t)=A x(t)+B v(t) \\
& y(t)=C x(t) \\
& C=\left[\begin{array}{cccccc}
0 & 0 & 0 & 1 & -l_{3} & l_{1} \\
0 & 0 & 0 & 1 & -l_{3} & -l_{2} \\
0 & 0 & 0 & 1 & l_{4} & -l_{2} \\
0 & 0 & 0 & 1 & l_{4} & l_{1}
\end{array}\right]
\end{aligned}
$$

여기서 $C$ 는 출력계수행렬이고, 출력방정식에는 센 서 다이나믹스를 포함하고 있다.

식 (4)의 모델에 대한 시뮬레이션을 통하여 얻은 주파수응답곡선을 시험결과와 함께 Fig. 3에 도시하 였다. Fig. 3에서 원 안의 고유진동는 $4.5 \mathrm{~Hz}$ 로서 이 것은 이후에 확인할 수 있듯이 수평방향 주파수성 분이 연성(coupled)되어 나타난 결과로 판단된다.

사각형 내의 공진점은 $8.5 \mathrm{~Hz}$ 와 $8.9 \mathrm{~Hz}$ 로 편심
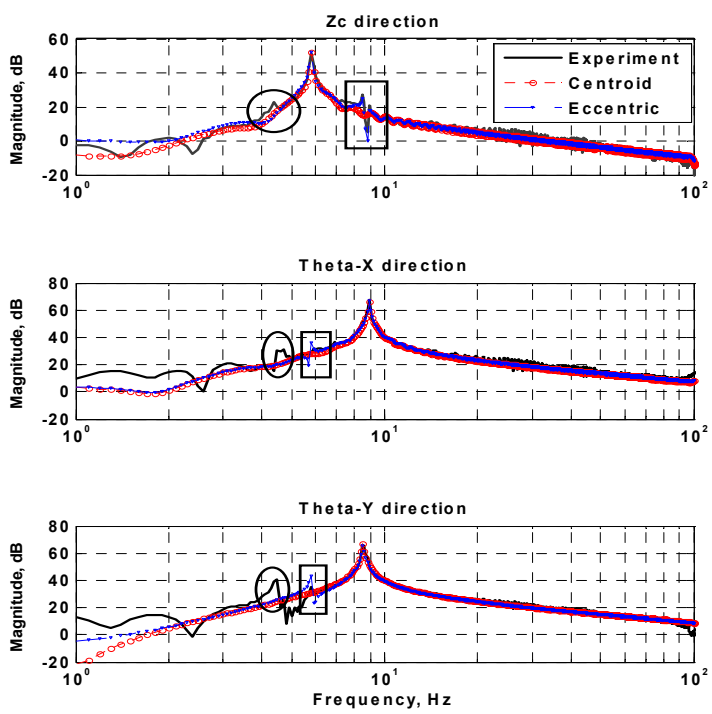

Fig. 3 Result of vertical frequency response test
으로 인한 영향이 각 자유도에 나타난 회전운동 성 분이다.

그리고 편심량은 약 $22 \mathrm{~mm}$ 로써 이격거리가 증가 할수록 사각부분의 크기가 증가한다. 하지만 시험결 과에서는 $\theta_{x}$ 및 $\theta_{y}$ 방향으로의 영향력이 거의 나타나 지 않았다. 따라서 이 연구에서 제진시스템은 질량 중심이 도심에 위치한다고 가정한다.

\section{2 수평방향 운동방정식}

수직방향에 대한 고찰 결과로부터 질량중심이 도 심에 있다는 가정하에서 수평방향에 대한 운동방정 식을 유도한다. Fig. 4는 수평방향 부품들의 배치 및 좌표기준을 나타낸다.

각 방향에 대한 변위를 $x_{c}(t), y_{c}(t), \theta_{z}(t)$ 로 정 의하고, 도심에서의 수평방향 변위에 대한 식으로 나타내면 다음과 같다.

$$
\begin{aligned}
& x_{c}(t)=\frac{x_{c 1}(t)+x_{c 2}(t)}{2} \\
& y_{c}(t)=\frac{y_{c 1}(t)+y_{c 2}(t)}{2} \\
& \theta_{z}(t)=\frac{x_{c 2}-x_{c 1}}{l y}=\frac{y_{c 2}-y_{c 1}}{l x}
\end{aligned}
$$

바닥에서 전달된 외란입력 $d_{h}(t)$ 으로 인하여 발 생한 상부 플레이트의 운동방정식은 식 (6)과 같다.

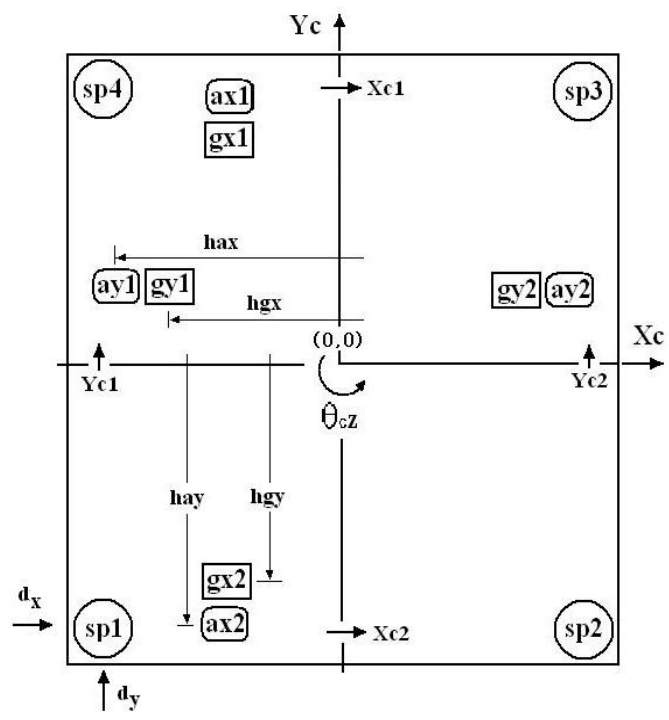

Fig. 4 Layout of horizontal components 


$$
\begin{aligned}
& M_{h} \ddot{\overline{x_{h}}}+D_{h} \dot{\overline{x_{h}}}+K_{h} \bar{x}_{h} \\
& =B_{h} v_{h}(t)+F_{h d} d_{h}(t)+F_{h d d} \dot{d}_{h}(t) \\
& M_{h}=\left[\begin{array}{ccc}
m & 0 & 0 \\
0 & m & 0 \\
0 & 0 & I_{z}
\end{array}\right] \\
& D_{h}=\left[\begin{array}{ccc}
2 c_{h x} & 0 & 0 \\
0 & 2 c_{h y} & 0 \\
0 & 0 & \left(c_{h x} l_{y}^{2}+c_{h y} l_{x}^{2}\right)
\end{array}\right] \\
& K_{h}=\left[\begin{array}{ccc}
2 k_{h x} & 0 & 0 \\
0 & 2 k_{h y} & 0 \\
0 & 0 & \left(k_{h x} l_{y}^{2}+k_{h y} l_{x}^{2}\right)
\end{array}\right] \\
& B_{h}=\left[\begin{array}{cccc}
\frac{k_{f x 1}}{R_{x 1}} & \frac{k_{f x 2}}{R_{x 2}} & 0 & 0 \\
0 & 0 & \frac{k_{f y 1}}{R_{y 1}} & \frac{k_{f y 2}}{R_{y 2}} \\
-\frac{k_{f x 1} h_{a y}}{R_{x 1}} & \frac{k_{f x 2} h_{a y}}{R_{x 2}}-\frac{k_{f y 1} h_{a x}}{R_{y 1}} & \frac{k_{f y 2} h_{a x}}{R_{y 2}}
\end{array}\right] \\
& F_{h d}=\left[\begin{array}{cccc}
k_{x} & k_{x} & 0 & 0 \\
0 & 0 & k_{y} & k_{y} \\
-s_{y} k_{x} & s_{y} k_{x} & -s_{x} k_{y} & s_{x} k_{y}
\end{array}\right] \\
& F_{h d d}=\left[\begin{array}{cccc}
c_{x} & c_{x} & 0 & 0 \\
0 & 0 & c_{y} & c_{y} \\
-s_{y} c_{x} & s_{y} c_{x} & -s_{x} c_{y} & s_{x} c_{y}
\end{array}\right]
\end{aligned}
$$

외란이 없는 경우에 대하여 수직방향에서와 마찬 가지로 동일한 절차에 따라 실시한 주파수응답시험 결과로부터 파라미터 값들을 추정하면 Table 2와 같 다. 여기서 스프링 강성 및 감쇠계수의 값이 수직방 향에 비해 크게 평가되었다.

$$
\left[x_{h 1}, x_{h 2}, x_{h 3}, x_{h 4}, x_{h 5}, x_{h 6}\right]^{T}=\left[x_{c}, y_{c}, \theta_{z}, \dot{x}_{c}, \dot{y}_{c}, \dot{\theta}_{z}\right]^{T} \text { 와 }
$$

Table 2 Estimated parameter values for horizontal direction

\begin{tabular}{c|c|c|c}
\hline \hline Parameter & Value & Parameter & Value \\
\hline$c_{x}$ & $7[\mathrm{Ns} / \mathrm{m}]$ & $c_{y}$ & $7[\mathrm{Ns} / \mathrm{m}]$ \\
\hline$f_{n x}$ & $4.5[\mathrm{~Hz}]$ & $f_{n y}$ & $4.7[\mathrm{~Hz}]$ \\
\hline$k_{x}$ & $8993.7[\mathrm{~N} / \mathrm{m}]$ & $k_{y}$ & $9810.9[\mathrm{~N} / \mathrm{m}]$ \\
\hline$k_{f x}$ & $3.6[\mathrm{~N} / \mathrm{V}]$ & $k_{f y}$ & $3.6[\mathrm{~N} / \mathrm{V}]$ \\
\hline$I_{z}$ & $2.3923\left[\mathrm{kgm}^{2}\right]$ & $f_{\theta z}$ & $6.95[\mathrm{~Hz}]$ \\
\hline$c_{z}$ & $1.6982[\mathrm{Ns} / \mathrm{m}]$ & $k_{z}$ & $4521.9[\mathrm{~N} / \mathrm{m}]$ \\
\hline$R_{x}$ & $16.6[\Omega]$ & $R_{y}$ & $16.6[\Omega]$ \\
\hline
\end{tabular}

같이 상태변수를 정의하고 식 (6)에 대하여 상태공 간 표현식을 유도하면 아래와 같다.

$$
\begin{aligned}
& \dot{x}_{h}(t)=A_{h} x_{h}(t)+B_{h} v_{h}(t) \\
& y_{h}(t)=C_{h} x_{h}(t)
\end{aligned}
$$

Table 2의 값들을 적용한 시뮬레이션 결과를 시 험결과와 함께 Fig. 5 에 도시하였다.

Fig. 5에서 볼 수 있듯이 $\mathrm{x}$ 및 $\mathrm{y}$ 축방향 직선운동 (swaying, surging)에는 $\theta_{z}$ 방향 성분(yawing)이 6.9 $\mathrm{Hz}$ 에서 동일하게 나타나고 있는 반면 swaying에는 rolling 성분이, surging에는 pitching 성분이 각각 영향을 끼치고 있다. 그런데 진폭의 크기가 수직방 향에서와는 달리 비교적 크게 나타나고 있어 향후 제어성능 구현 시 외란입력으로 작용할 가능성이 있다. 이에 대한 영향은 실제 제어시험을 통해 확인 하고자 한다. 한편 yawing 운동에서는 다른 운동모 드의 영향이 상대적으로 작게 나타나고 있다.

\section{4 제진성능 구현}

제어기 설계는 식 (3)과 (5) 모델에 대하여 앞서 추정한 파라미터 값들을 적용하고, loop shaping 기 법을 응용하였다. 이 제어기 설계방법은 루프 전달 함수 $\left(L(s)=G_{\text {plant }}(s) G_{\text {controller }}(s)\right)$ 의 주파수응답 특 성으로부터 원하는 폐루프 응답특성을 만족시키는 기법으로 매우 실용적이다.

Loop shaping 설계기법의 주요 설계사양은 아래 와 같다 ${ }^{(9)}$.

감도함수(sensitivity function) : 제어 주파수 영역 내에서 가능한 한 작아야 한다. 이를 위해서는 $|L(s)|$ 의 값이 가능한 한 크도록 설계 한다.

교차주파수 인근 사양: 이득여유(gain margin)와 위상여유(phase margin) 설계사양으로 폐루프 시스 템의 안정성을 확보한다.

Feedforward 제어입력은 geophone 센서의 저주 파대역의 응답특성을 보상하도록 디지털 필터를 적 용하여 구현하였다.

성능시험은 무부하 상태에서 시스템 주변을 걷거 나, 점프하는 등의 암진동을 주며 실시하였다.

Fig. 6에 수동 및 능동상태에서의 전달률(transmissibility) 곡선을 함께 나타내었다. 전달률은 바닥 

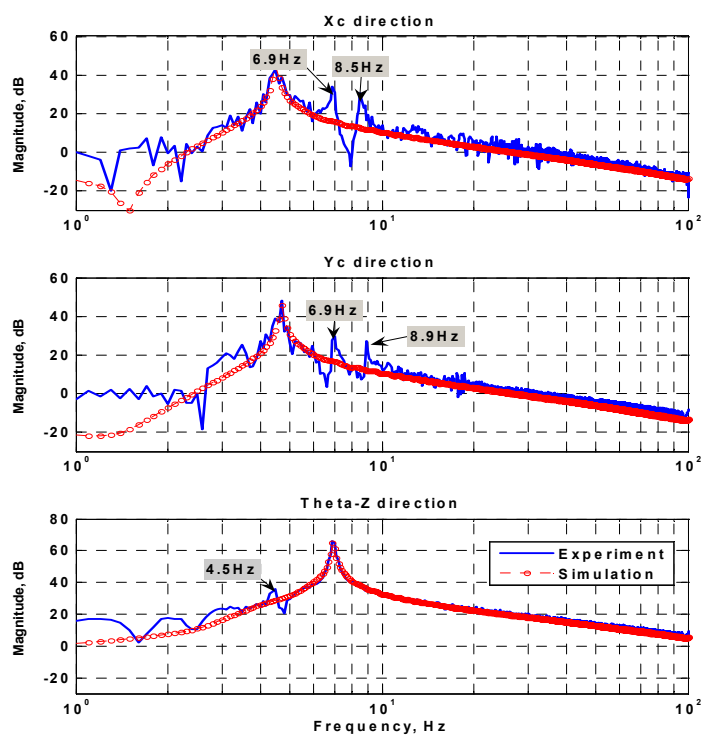

Fig. 5 Result of horizontal frequency response test
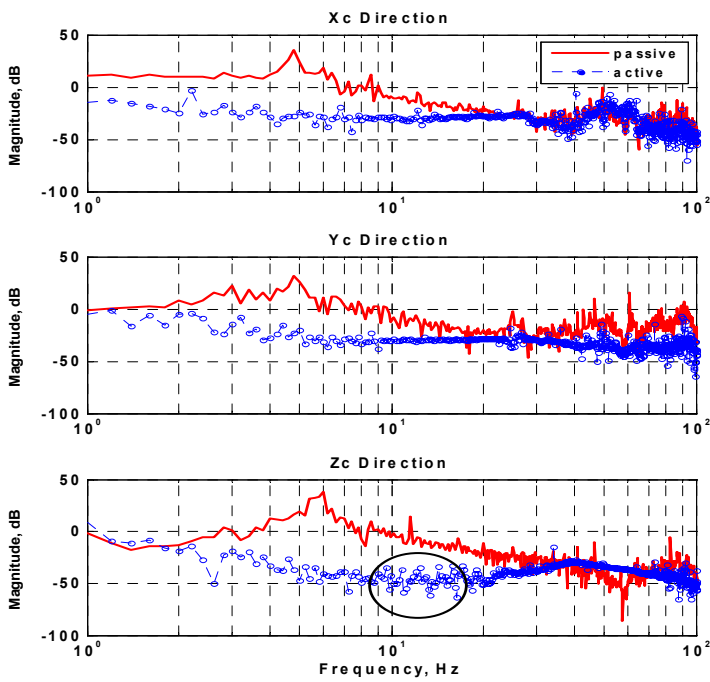

Fig. 6 Transmissibility curves of the vibration isolation system

외란 입력에 대한 상부 플레이트 출력신호의 비 (ratio)로 정의하였으며, 시스템에 내장된 속도센서 로부터 얻은 신호를 $\mathrm{FFT}$ (fast fourier transform)처 리하여 데시벨 $(\mathrm{dB})$ 값으로 나타내었다.

그림에서 실선은 수동형, 원 마크가 있는 선은 능 동형 시스템의 성능곡선으로 수직방향은 roll-off rate $-40 \mathrm{~dB} / 10 \mathrm{~Hz}$ 를 만족시키는 반면 수평방향으로는 -30 $\mathrm{dB}$ 정도로 다소 부족하게 나타났다. 한편 앞서 언급
했던 rolling 및 pitching 성분의 연성효과는 유효하 게 제어되고 있는 것으로 나타났다. 제진 성능이 부 족하게 나타난 이유는 제어력 부족에 기인한 것으 로 판단된다. 한편 그림의 원부분과 같이 전달률 곡 선이 매끄럽지 않은 이유는 두 신호사이의 코히어 런스(coherence)가 좋지 않은 결과이다. 그것은 가 진방법에 크게 의존하기 때문에 깨끗한 그래프를 얻기 위해서는 가진시스템이 올바르게 구비된 장비 위에서 평가가 이루어져야 할 것이다.

\section{5. 결 론}

$\mathrm{VCM}$ 을 사용한 능동형 제진시스템에 대하여 수 직방향에 대한 편심상태에서의 모델링을 수행하고, 시뮬레이션 및 주파수응답시험을 통하여 응답특성 을 비교한 결과 편심의 정도 및 영향이 크지 않은 것으로 확인되었다. 따라서 질량중심이 도심에 있는 경우에 대하여 수평방향 모델링을 수행하여 6자유 도 시스템에 대한 수학적 모델을 완성하였다. 그리 고 모델링 과정에서 추정한 파라미터 값들을 적용 하고, loop-shaping 기법 및 feedforward 제어기법 을 응용하여 수직방향으로 $-40 \mathrm{~dB} / 10 \mathrm{~Hz}$ 의 원하는 제진성능 목표을 달성하였다.

향후의 연구는 수평방향 성능개선과 같이 보다 세밀한 제어성능 개선문제 및 에너지소비 최소화를 위한 제어입력 생성방법 등에 대하여 진행하고자 한다.

\section{참 고 문 헌}

(1) Cho, J. H., Lee, J. W., Lee, C. K. and Kwon, B. I., 2004, "Optimal Design of Extremely Small Thrust VCM for Nanoindenter," Transactions of the Korean Institute of Electrical Engineers, Vol. 53B, No. 2, pp. 69 74.

(2) Lee, H. K., You, Y. M. and Kwon, B. I., 2004, "Design and Characteristic Analysis of a Cylinderical Voice Coil Motor," Proceedings of the KIEE Annual Summer Conference, pp. 1034 1036.

(3) Choi, K. B., Park, K. W., Kim, S. H. and Kwak, Y. K., 1996, "Development of a Noncontacting 6 DOf Micro-positioner Driven by 
Magnetic Force -Design, Modeling and Control-," Transactions of the Korean Society of Mechanical Engineers, Vol. 20, No. 4, pp. 1164 1176.

(4) Muller, T., Hurlebaus, S., Stobener, U. and Gaul, L., 2005, "Modeling and Control Techniques of an Active Vibration Isolation System," Proceedings of the IMAC XXIII.

(5) Kim, H. S., Moon, J. H., Park, H. J. and Cho, Y. M., 2006, "Uncertainties and Control of a 3-DOF Active Vibration Isolation System," Transactions of the Korean Society for Noise and Vibration Engineering, Vol. 16, No. 12, pp. 1262 1271.

(6) Zuo, L. and Nayfeh, S. A., 2004, "An Active-passive Stage for Vibration Isolation and
Positioning Control," Proceedings of the 7th International Conference on Motion and Vibration Control, pp. 643 648.

(7) Son, T. K., Kim, K. Y., You, W. H. and Park, Y. P., 1994, “A Study on Active Vibration Isolation Using Electro-magnetic Actuator," Transactions of the Korean Society of Mechanical Engineers, Vol. 18, No. 5, pp. 1169 1181.

(8) Fuller, C. R., 1996, Active Control of Vibration, Academic Press, Inc., New York.

(9) Barratt, C. and Boyd, S., 1992, "Interactive Loop-shaping Design of MIMO Controllers," Proceedings of IEEE Symposium on Computer Aided Control System Desing, pp. 76 81. 